\title{
Perioperative Issues in Sleep Disordered Breathing
}

\section{A. G. Ghoshal, Indranil Halder}

See end of article for authors

Indian J Sleep Med 2006; 1.2, 81-86

affiliations

Address for correspondence:

Aloke Gopal Ghoshal

Head of the Department

Department of Respiratory Medicine

NRS Medical College, Kolkata

Keywords : obstructive sleep apnoea, perioperative issues, airway management

\section{Intoduction}

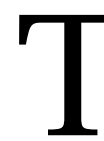

he estimated prevalence of obstructive sleep apnoea in middle age is $2 \%$ for women and $4 \%$ for men. ${ }^{1}$ Approximately $9 \%$ of women and $24 \%$ of men aged 30 to 60 have an apnoea / hypopnea index of $\geq 5 .{ }^{2} \mathrm{H}$ owever, obstructive sleep apnoea (OSA) is undiagnosed in a large section of the population. According to different estimates $80 \%$ to $90 \%$ of patients with OSA are undiagnosed. ${ }^{1,3}$ D iagnosed OSA patients, as well as undiagnosed patients who may not present with classic signs and symptoms, areat risk for significant post-operative respiratory complications. This relates to all the stages a patient goes through for having an operation - preoperative makeup, general anaesthesia, intubation and extubation, and postoperative analgesia and vigil. Just simple screening for patients with a diagnosis of OSA misses most of the sufferers. Even diagnosed OSA patients, most of the times, fail to get the attention and planned approach needed for their perioperative management, which increases their morbidity and mortality.

\section{Pathophysiology}

In a normal airway, the negative pressure created by inspiraton is counteracted by thetone of the upper airway musculature thereby maintaining airway patency. The electrical activity within upper airway muscle, particularly the genioglossus and tensor palatini, gets reduced after the onset of sleep, making collapse of the pharyngeal walls more likely. This tendency is compounded by consuming alcohol or other sedatives. D uring rapid eye movement sleep in particular, the body is at its most relaxed state, and a three dimensional collapse of muscle and fatty tissue around the upper airway may cause obstruction. ${ }^{4}$ W hen a pre-existent narrowing and slackening of the upper airway is also present ${ }^{5}$ apnoeas (complete cessation of breathing for 10 seconds or more) or hypopnoeas ( $>50 \%$ diminishing of airflow or oxygen desaturations $>3 \%$ for 10 seconds or more) may result. Patients with obstructive sleep apnoea are particularly vulnerable during anaesthesia and sedation. ${ }^{6}$ W hen a patient known to have sleep apnea is treated surgically, the patient is particularly at risk because of the effect of anesthesia both on the control of the respiration and on the tone of the upper airway musculature. Anesthetic, opiate, and sedative agents are central nervous system (CN S) depressants that increase the tendency for upper airway collapse. Additionally, CN S depressants alter the normal ventilatory response to hypercapnia and hypoxemia. ${ }^{7}$ So, CN S depressants acts in both ways. Firstly it decreases pharengeal muscle tone and secondly it decreases neural stimulus to breath. The decreased pharyngeal muscle tone in turn causes partial airway obstruction, which results in hypopnea and obstructive apnea. The decreased neural stimulus to breathe causes shallow respiration and hypoxia and central apnea. Both CN S effects may be significantly worse in OSA patients as the decreased arousal response to breathe leads to 
prolonged apnea and the potential for respiratory and cardiovascular arrest., ${ }^{8}$ Compounding this problem is the technical difficulty of managing the airway, of bag and mask ventilation, and of endotracheal intubation, particularly in a crisis situation on a medical/surgical ward.

\section{The problem}

There are two major concerns. First, the airway. The existing narrowing and increased likelihood of morphological alterations of the upper airway lead to increased difficulties in securing and maintaining a patent airway. Secondly, use of sedation and narcotics needs close attention since these patients tend to be extremely sensitive to even small doses of them. Even after surgery not related to obstructive sleep apnea, such as hip and knee operations, patients with obstructive sleep apnea areat risk of developing respiratory and cardiopulmonary complications postoperatively. Serious complications include reintubations and cardiac events and emergency reintubation is almost extremely difficult. ${ }^{10}$

\section{Recognition of the problem}

Early recognition of undiagnosed obstructive sleep apnoea, at preoperative screening is essential. $\mathrm{H}$ eavy and persistent snoring, sudden awakenings accompanied by choking, apnoeas as observed by the bed partner, and excessive sleepiness during daytime are, as we all know, characteristic symptoms of obstructive sleep apnoea. ${ }^{11}$ But obstructivesleep apnoea can also be associated with a wide variety of other symptoms and signs, which unfortunately arenot very specific. TheEpworth sleepiness scale can be used to screen for obstructive sleep apnoea, but its specificity and sensitivity are also low. Ideally, full night polysomnography is used to determine if obstructive sleep apnoea is present. 0 besity (body mass index $>30$ ) and especially a large neck circumference $(>44 \mathrm{~cm})$ have a positive correlation with severe obstructive sleep apnoea, because these conditions involve extensive soft tissue enlargements of the upper airway. Other predisposing factors include increasing age, male sex, and use of alcohol. ${ }^{12,13}$

It is not sufficient to simply ask if the patient has sleep apnea or disturbance. Physical characteristics associated with obstructive sleep apnoea have to be looked for (Table 3). The risk of developing perioperative complications is increased not only by the presence of obstructive sleep apnoea but also, the co morbidities. The co-morbidities include cardiovascular ${ }^{14}$, which includes hypertension, cardiomyopathy, venous stasis diseases, deep vein thrombosis, pulmonary hypertension and right sided heart failure. The pulmonary co morbidities include COPD, hypoventilation syndrome of obesity and asthma. The metabolic co morbidities include Type II Diabetes, hyperlipidemia, hypercholesterolemia and $\mathrm{N}$ onalcoholic steatotic hepatits. The gastrointestinal co morbidities includes gastroesophageal reflux disease and cholelithiasis. The musculoskeletal co morbiditiesinclude degenerativejoint disease, lumber disc disease, osteoarthritis and ventral hernias. A previous sleep study, if available, may help. If no sleep study has ever been conducted - or if one has been conducted before significant weight gain or another current potentially associated factors - a sleep study may be warranted. In cases of planned surgery- a gold standard is full overnight polysomnagraphy. All non-

Table 1

Symptoms associated with 0 bstructive sleep apnoea in Adults

H eavy persistent snoring

- Excessive daytime sleepiness

- Apnoeas as observed by bed partner

- Choking sensations while waking up

- G astro-oesophageal reflux

- Reduced ability to concentrate-M emory loss

- Personality changes

- M ood swings

- N ight sweating

- N octuria

- D ry mouth in the morning

- Restless sleep

- M orning headache

- Impotence
Symptoms associated with obstructive sleep apnoea in Children

- Snoring

- Restless sleep. Sleepiness

- $\mathrm{H}$ yperactivity

- Aggression and behavioral disturbance

- Frequent colds or coughing

- O dd sleeping position 
emergent surgery may be postponed to allow investigatons and if necessary to start treatment with CPAP.

of particular importance is any previous history of anesthesia or surgery. Records should be reviewed for information pertaining to the anesthetic technique employed or any adverse intraoperative or postoperative events.

\section{Patient Communication}

The patient should be informed about the increased risk presented by surgery and anesthesia, including the need for awake intubation, the possibility of postoperative intubation and mechanical ventilation, and the possibility of a tracheostomy if attempts at airway management are unsuccesful. For patients who are al ready on CPAP the treatment should be continued until the morning of operation and the patient asked to bring the CPAP instrument in hospital and it is crucial to maintain CPAP after surgery. It is also mandatory to check the performance of the patient's CPAP machine.

\section{Assessment of risk that tracheal intubation may be difficult}

Difficult intubation is found in $15-40 \%$ of patients of OSA, compared with $1-3 \%$ in normal population. $O$ besity is a significant risk factor

Alterations in craniofacial morphology contributing to obstructive sleep apnoea ${ }^{15}$ - such as macroglossia, retrognathia, a narrow hypopharynx because of fat deposition in the lateral walls of the pharynx, or an anteriorly displaced larynx have to be looked for. $M$ allampati ${ }^{15}$ constructed a staging system to predict difficult tracheal intubation, and this system was modified by Friedman to enable estimation of the success rate of uvulopalatopharyngoplastyat the sametime. The position of the tongue is assessed in relation to that of the soft palate and, depending on the size of the tongue, four stages can be distinguished. In general, oral intubation is hindered when patients are categorised as $M$ allampati stage 3 or 4, although different investigators have failed to find a correlation of this preoperative staging system with more difficult intubation.

Any patient diagnosed as having obstructive sleep apnoea or in whom it is suspected on the basis of clinical signs (obesity, limited mouth opening, or a large tongue) should betreated as having a difficult ai rway until proved
Table 3

Physical characteristics associated with obstructive sleep apnoea

- $\mathrm{N}$ asal obstruction (deviation of the septum or hypertrophic conchae inferiores)

- O edematous or long soft palateor uvula. $\mathrm{H}$ ypertrophic tongue tonsils

- N arrow oropharynx (large tonsils, redundant pharyngeal arches)

- Adiposity or large neck circumferenceRetrognathia

- M axillary hypoplasia

otherwise. O rotracheal intubation, especially in severely obese patients with obstructive sleep apnoea, may be extremely difficult, and postoperativeairway obstruction due to swelling or bleeding should be anticipated. Of course, no staging system can prevent unexpected difficulties in tracheal intubation.

$M$ any patients with OSA are morbidly obese (e.g.: more than two times of ideal body weight). This places them at increased risk for, among other things, aspiration of acidic gastric fluid at the time of induction of anesthesia. It is for this reason that many of these patients receive medication to suppress gastric acid production, to neutralize the acid, or to stimulate emptying of stomach.

0 ther potential preoperative challenges with patients who are obese can include obtaining adequate reliable

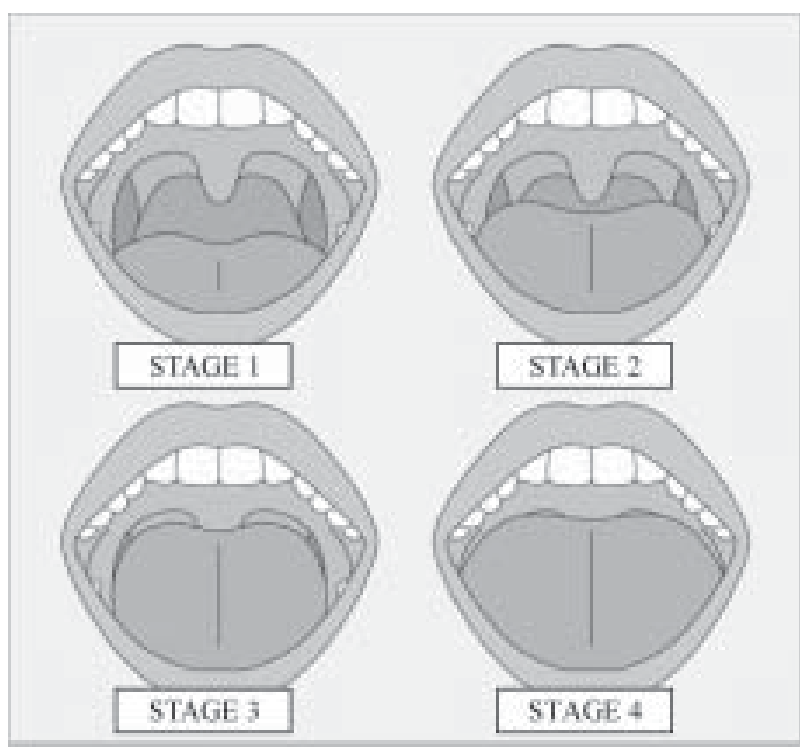

Indian Journal of Sleep M edicine (IJSM), Vol. 1, N o. 2, 2006 
intravenous access.

\section{Premedication}

Preoperative sedation with anticonvulsive and muscle relaxing effects on the upper airway musculature may cause an appreciable reduction of the pharyngeal space. Consequently, a higher risk of preoperative phases of hypopnoea and consecutive hypoxia and hypercapnia arises after administration, and oxygen saturation needs to bemonitored adequately. ${ }^{16}$ An effectiveanxiolytic agent will reduce the dose of anaesthetic needed to induce general anaesthesia, which may otherwise lead to an increased likelihood of cardiovascular complications. If needed, oxygen can be given by an insufflation mask preoperatively, and application of nasal continuous positive airway pressure might be necessary postoperatively.

Even minimal sedation can cause airway obstruction and ventilatory arrest. Therefore, many anesthesiologists do not favour preoperative sedatives to patients with OSA.

\section{Intubation technique}

The main goal in all patients is to avoid inadequate ventilation and oxygenation. This may result in increased morbidity and mortality due to hypoxaemia or hypercapnia and associated haemodynamic changes (such as tachycardia, arrhythmia, and hypertension). The adverse events may include damage to teeth, airway trauma, cardiopulmonary arrest, brain injury and death.

So the consensus is to intubate these patients awake, ${ }^{17}$ with or without using a fiberoptic laryngoscope and to have an experienced surgeon available in the operating room at the time of induction of general anesthesia in case of tracheostomy becomes necessary.

Regardless of the primary anesthetic technique chosen, airway maintenance, especially with sedation, remains a fundamental concern to the anesthesiologist.

0 xygen must be administered for three or more minutes before intubation and, whenever possible.

The period of awakening from anesthesia can be problematic for patients with OSA. In patients who have just undergone surgery for the treatment of their OSA, the airway can be narrowed from swelling and inflammation. Also, lingering sedative and ventilatory depressant effects of the anesthetic can pose difficulty. Perioperative vigilance should continue into the postoperative period.

$M$ any patients require postoperative intubation and mechanical ventilation until fully awake. A CPAP (Continuous Positive Airway Pressure) machine can be employed in some patients postoperatively to support breathing. For certain patients, it may be prudent to admit them to an immediate care or intensive care area postoperatively to facilitate close monitoring and airway support measures.

\section{Extubation}

Tracheal extubation should be carried out only when the patient is conscious, communicative, and breathing spontaneously with an adequate tidal volume and oxygenation. A half sitting or Trendelenberg position is preferable. $^{18}$

Some prefer a single bolus of methylprednisoloneafter theinduction of the anesthesia to help in avoiding swelling of the pharynx after surgery. ${ }^{18}$

\section{Post operative follow up}

Respiratory depression and repetitive apnoeas may occur directly after extubation in patients with obstructive sleep apnoea. ${ }^{6} \mathrm{~N}$ arcotic intravenous administration may cause delayed (4-12 hours after administration) respiratory depression. $\mathrm{N}$ arcotic should only be used when nonsteroid anti-inflammatory drugs or regional anaesthesia cannot be administered or is insufficient. In some cases it may be wise to titrate short acting opioid variants until pain sensation is sufficiently diminished. The patient needs to be observed closely, preferably in an intensive care unit.

O stermeier et al state that patients with obstructive sleep apnoea are at increased risk of developing respiratory problems postoperatively in the absence of pain. Pain would prevent therebound of rapid eye movement sleep and diminish stage 3 and stage 4 sleep, which also predisposes to collapse of the upper airway, around the third day postoperatively. ${ }^{19}$ The risk of developing respiratory depression can be reduced by the use of nasal continuous positive airway pressure preoperatively and directly postoperatively.

Surgery of the upper airway- especially 
uvulopalatopharyngoplasty and (adeno)tonsillectomy causes tissue damage and produces oedema and occasionally (considerable) haematomas which may increase narrowing of the upper airway. These may lead temporarily to severe obstructive sleep apnoea, particularly when opioids and sedating drugs are administered. M ortality after reconstruction of the soft palate (palatoschisis), ${ }^{20}$ due to obstruction of the upper airway, has been reported. Prolonged tracheal intubation or tracheotomy may be needed in patients with severe obstructive sleep apnoea.

$\mathrm{N}$ asal or sinus surgery with use of packs constitutes an extra risk for obstructive sleep apnoea. O bservation in a medium or high care unit as long as the packs are in place is advisable, and in the case of severe obstructive sleep apnoea full facecontinuous positive ai rway pressure is needed to prevent dangerous apnoeas. A ventilation channel inside the nasal pack, a therapeutic alternative is often very disappointing.

The patient's nose should be examined frequently for areas of pressure necrosis if the mask is worn for prolonged periods of time. As prophylaxis, the patient should have more than one mask of slightly different sizes so that there are two different pressure points.

Patients who have had a sternotomy or thoracotomy may complain of severe chest wall pain in the morning after they have been on CPAP. This might be related to the stretching of the incision by the increased intrathoracic pressure.

\section{Condusion}

Patients with obstructive sleep apnoea are at high risk of developing complications when having surgery or other invasive interventions under general anaesthesia, whether or not the surgery is related to obstructive sleep apnoea. ${ }^{21}$

Physicians, in addition to surgeons of all specialties and anaesthetists, should befamiliar with theseproblems. ${ }^{21}$

$M$ anagement options include awake intubation and extubation, alternative methods of pain relief, use of nasal continuousairway pressure before and after surgery, and surveillance in an intensive care unit if needed. $M$ anagement decisions should be a team effort with the physician, surgeon and anesthesiologist.

\section{Authors' affiliations \\ Prof. Aloke Gopal Ghoshal \\ Head of the Department \\ Department of Respiratory Medicine \\ NRS MEDICAL COLLEGE, Kolkata.}

\section{References}

1. Young T, Evans L, Finn L, Palta M. Estimation of the clinically diagnosed proportion of sleep apnea syndrome in middleaged men and women. Sleep 1997; 20: 705-6.

2. Young T, Palta M, Demsey J, et al. The occurrence of sleep disordered breathing among middle aged adults. N Engl J Med. 1993; 328:1230-1235.

3. Young T, Evan L, Finn L, et al. Estimation of the clinically diagnosed proportion of sleep apnea syndrome in middle age men and women. Sleep. 1997; 20:705-706.

4. Strohl KP, Cherniack NS, Gothe B. Physiologic basis of therapy for sleep apnea. Am Rev Respir Dis 1986;134: 791-802

5. Gleadhill IC, Schwartz AR, Schubert N, Wise RA, Permutt $\mathrm{S}$, Smith PL. Upper airway collapsibility in snorers and in patients with obstructive hypopnea and apnea. Am Rev Respir Dis 1991; 143: 1300-3.

6. Loadsman JA, Hillman DR. Anaesthesia and sleep apnoea. $\mathrm{Br}$ J Anaesth 2001; 86: 254-66.

7. Cullen DJ. Obstructive sleep apnea and postoperative analgesia-a potentially dangerous combination. J Clin Anesth. 2001; 13:83-85.

8. Loadsman JA, Hillman JR. Anesthesia and sleep apnoea. Br J Anaesth. 2001; 86:256-254.

9. Robinson RW, Zwillich CW, Bixler EO, et al. Effects of oral narcotics on sleep disordered breathing in healthy adults. Chest. 1987; 91:197-203.

10. Gupta RM, Parvizi J, Hanssen AD, Gay PC. Postoperative complications in patients with obstructive sleep apnea syndrome undergoing hip or knee replacement: a casecontrol study. Mayo Clin Proc 2001; 76: 897-905.

11. Reimer MA, Flemons WW. Q uality of life in sleep disorders. Sleep Med Rev 2003; 7: 335-49.

12. Young T, Palta M, Dempsey J, Skatrud J, Weber S, Badr S. The occurrence of sleep disordered breathing among middle-aged adults. N Engl J Med 1993; 328: 1230-5.

13. Barthel SW, Strome M. Snoring, obstructive sleep apnea and surgery. Med Clin North Am 1999;83: 85-96.

14. Shahar E, Witney CW, Redline S, Lee ET, Newman AB, Javier Nieto $F$, et al. Sleep-disordered breathing and cardiovascular disease. Am J Respir Crit Care Med 2001; 163: 19-25.

15. Verin E, Tardif C, Buffet X, Marie JP, Lacoume Y, AndrieuGuitrancourt J, et al. Comparison between anatomy and resistance of upper airway in normal subjects, snorers and OSAS patients. Respir Physiol 2002; 129: 335-43.

Indian Journal of Sleep M edicine (IJSM ), Vol. 1, N o. 2, 2006 
16. Connolly LA. Anaesthetic management of obstructive sleep apnea patients. J Clin Anesth 1991; 3: 461-9.

17. Mallampati SR, Gatt SP, Gugino LD, Desai SP, Waraksa B, Freiberger $\mathrm{D}$, et al. A clinical sign to predict difficult tracheal intubation: a prospective study. Can Anaesth Soc J 1985; 32: 429-34.

18. Sheppard LM, Werkhaven J, Mickelson S, Crissman JD, Peterson E, Jacobsen G. Effect of steroids or tissue precooling on oedema and tissue thermal coagulation after CO 2 laser impact. Laser Surg Med 1992; 12: 137-46.
19. Knill RL, Moote CA, Skinner MI, Rose EA. Anesthesia with abdominal surgery lead to intense REM sleep during the first postoperative week. Anesthesiology 1990;73: 52-61.

20. Valnicek SM, Zuker RM, Halpern LM, Roy WL. Perioperative complications of superior pharyngeal flap surgery in children. Plast Reconstr Surg 1994; 93: 954-8.

21. Cindy den Herder, Joachim S, Dick J K A, Nico de Vries. Risks of general anaesthesia in people with obstructive sleep apnoea. BMJ 2004; 955 - 959. 